\title{
Path Feasibility and Modification
}

\author{
Kuu-young Young \\ Department of Control Engineering \\ National Chiao-Tung University \\ Hsinchu 30039, Taiwan, ROC \\ Chi-haur Wu \\ Department of Electrical Engineering and Computer Science \\ Northwestern University \\ Evanston, IL 60208
}

Received November 30, 1990; accepted July 26, 1991

Kinematic feasibility of a planned robot path is restrained by the kinematic constraints of the robot executing the task, such as workspace, configuration, and singularity. Since these kinematic constraints can be described utilizing the geometry of the given robot, corresponding regions within the robot workspace can be expressed in geometrical representation. Consequently, geometric information can be derived from the planned path and the geometric boundaries of these regions. Then, by utilizing the geometric information and proper modification strategies, a Cartesian robot path that is kinematically infeasible can be modified according to different task requirements. To demonstrate the proposed feasibility and modification schemes, simulations for a $6 \mathrm{R}$ robot manipulator are executed.

計画されたロボットのバスの逜䖝学的可能性は、タスクを実行するロボットのワ

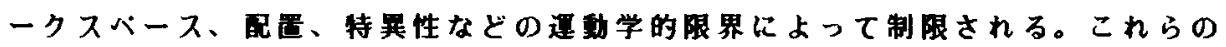
運動学的制限はロボットの幾何学梏造を利用して表現できるので、ロホットのワ

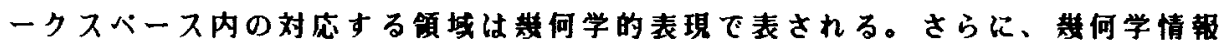
は、計画されたバスと幾何学的境界から竞かれる。これらの何学情抜と通切に 改良された方法を用いることで、遇動学的に不可能だったCartesian口ボットのハ スを異なる夕スクの要求に対して改良できる。提案の可能性と改良した手法の性 能を、6 Rロボット・マニピュレータでシミュレーションで確認している。

\section{INTRODUCTION}

As there is not enough information about different kinematic constraints in the existing planning stage, a planned Cartesian path by a Computer-aided design (CAD) system may not be kinematically feasible. Currently, a well-

Journal of Robotic Systems 9(5), 613-633 (1992).

(C) 1992 by John Wiley \& Sons, Inc.

CCC 0741-2223/92/050613-021\$04.00 
known and simple method to test whether a Cartesian path is within feasible regions of the robot workspace is to compute the inverse kinematic solutions.' Though the approach of the inverse kinematic solutions can be used to determine the feasibility of a given path, it is not suitable for providing the necessary geometric information for modification. Consequently, a detected infeasible path has to be replanned even if only a small portion of the path is infeasible. In addition, the replanned path may be still infeasible. Therefore, it will be beneficial to have proper representation of the geometric information related to the kinematic constraints, such as feasible regions, areas of different configurations, and singular areas. In other words, based on the geometry of the given robot, the robot workspace should be formulated and incorporated with the knowledge of the kinematic constraints such that path modification may be performed. Hence, a planned Cartesian path by a CAD system can be modified efficiently and the integration between $\mathrm{CAD}$ /robots be enhanced.

There are a lot of achievements in the analysis of workspace. Among them, Kumar and Waldron and Yang and Lai discussed the dexterous workspace, which is important in the analysis of the orientational workspace. ${ }^{2,3}$ Tsai and Sony dealt with the synthesis of robot arms for a prescribed path based on twoand three-link robots. ${ }^{4}$ Hansen et al. and Lee and Yang tackled the problems of how to generate the workspace. ${ }^{5,6}$ Litvin et al. derived the functions of singularities, configurations, and displacements for robot manipulators. ${ }^{7}$ And, Vijaykumar et al. developed criteria for optimizing 6R manipulator structures considering working volume and dexterity. ${ }^{8}$ To find suitable expressions to describe the workspace, in this article the proposed analysis on workspace will take advantage of the geometric approach in solving inverse kinematics by treating the workspace of a robot manipulator as a geometric object bounded by joints' limits the ranges. ${ }^{9}$ In other words, the kinematic arrangements of the given robot are utilized to describe the geometric boundaries of different regions corresponding to different kinematic constraints inside the robot workspace. ${ }^{10}$ Then the sets of joint variables specifying the planned path are mapped into the corresponding traces inside the robot workspace. Based on these geometric expressions, the geometric information describing the relationship between the planned path and the kinematic constraints can be easily derived. Consequently, the kinematic feasibility testing and modification can be achieved.

To perform path modification, proper modification strategies are also needed. In general, modification strategies depend on the applications and user's specifications. It means that the requirements, consequently the freedoms available for modification, will determine the modification strategies. For instance, if the task is to move a directionless object from one to another location, then only the starting and end positions need to be considered and the intermediate positions and orientations may be arbitrary. If the object to move is a cup of coffee, then the orientation specification is also of concern. As a result, with the geometric information and proper modification strategies, an infeasible path can be modified accordingly.

For covering the variety of industrial robot manipulators, the workspaces of 
the robot manipulators consisting of combinations of different types of joints with offsets in between are analyzed. The analysis will concentrate on nonredundant wrist-partitioned types of robot manipulators. To demonstrate the proposed path feasibility and modification schemes, simulations for a $6 \mathrm{R}$ robot manipulator are executed.

\section{WORKSPACE ANALYSIS}

Distinguished by position and orientation, the workspace analysis can be separated into two parts: positional and orientational workspaces. Thus, a planned path is said to be feasible if its corresponding traces are within feasible regions of the positional and orientational workspaces. Since there are no link lengths among the minor joints of the wrist-partitioned type of robot manipulator, it is proposed to choose the wrist position $\mathbf{p}_{w}$ as the reference point. Then the positional workspace can be defined as that of the wrist constrained by the primary joints, denoted as $P W K$ :

$$
P W K=\left[\mathbf{p}_{w} \mid \mathbf{p}_{w}=\mathbf{f}\left(\boldsymbol{\theta}_{p}\right) \text { for every } \boldsymbol{\theta}_{p}\right]
$$

where $\boldsymbol{\theta}_{p}$ denotes a set of joint variables of the primary joints and $\mathbf{f}$ is a forward kinematic function, which transforms a set of joint variables into its corresponding Cartesian position. Inside $P W K$, the subworkspace corresponding to feasible regions, $F P W K$, can be specified via the similar transformation as in eq. (1):

$$
F P W K=\left[\mathbf{p}_{w} \mid \mathbf{p}_{w}=\mathbf{f}\left(\boldsymbol{\theta}_{p}\right) \text { for every feasible } \boldsymbol{\theta}_{p}\right]
$$

where feasible $\boldsymbol{\theta}_{p}$ means the elements of $\boldsymbol{\theta}_{p}$ are within the joint limits. Following the same formulation, the subworkspaces corresponding to different kinematic constraints, such as areas of different configurations and singular areas, can also be determined. Here, the areas of different configurations are defined as the areas corresponding to various arm configurations due to the special geometry of the given robot, and the singular areas as the vicinities of the boundaries of the feasible regions and positions where the corresponding joint variables are undefined. With the robot workspace described in these expressions, the planned path can be identified inside the workspace by mapping the sets of joint variables specifying the planned path into the corresponding traces. Via this geometrical approach, the mapping transforms the analysis of joint variables in numerical representation into traces in the workspace in geometrical representation. As a result, the geometric information related to the kinematic constraints and neighboring points in these workspaces can be easily derived.

It can be seen that each element within $P W K$ will not only determine a wrist point but also a wrist coordinate frame for mounting the minor joints. Thus, the orientational workspace $O W K$ consists of two parts: the orientational workspace of the wrist as the function of the primary joints $O W K^{p}$ and the orienta- 
tional workspace at the tool tip, with respect of $O W K^{p}$, as the function of the minor joints $O W K^{m}$. They can be represented as:

$$
O W K^{p}=\left[R_{w} \mid R_{w}=\mathbf{g}\left(\boldsymbol{\theta}_{p}\right) \text { for every } \boldsymbol{\theta}_{p}\right]
$$

where $R_{w}$ denotes the orientational part of a wrist point and $\mathbf{g}$ is a forward kinematic function, which transforms a set of joint variables into its corresponding orientation, and

$$
O W K^{m}=\left[{ }^{w} R_{e} \mid{ }^{w} R_{e}=\mathbf{g}\left(\theta_{m}\right) \text { for every } \boldsymbol{\theta}_{m}\right]
$$

where ${ }^{w} R_{e}$ denotes the orientational part of the end effector with respect to the wrist coordinates and $\boldsymbol{\theta}_{m}$ a set of joint variables of the minor joints. Similarly, the feasible regions, areas of different configurations, and the singular areas can be identified within $O W K$.

To analyze these workspaces, the number of the primary and minor joints is assumed to be three for each, which will provide 6 degrees of freedoms (dof). Those types with less dof can be taken as the subset of the discussed cases. With 3 dof, there can be at most eight combinations of revolute (R) and prismatic (P) types of joints with offsets in between for the primary joints, summarized in Table $\mathrm{I} .{ }^{11}$ As for the minor joints, since the discussed robot manipulators are wrist-partitioned there will be no offsets assigned to them and they consist of all revolute joints.

\subsection{Positional Workspace}

To analyze $P W K$, the wrist position $\mathbf{p}_{w}$ is employed. In general, a Cartesian path of a 6 dof robot manipulator can be specified by a homogeneous transformation $P O S$ on a point-by-point basis, and $P O S=B A S E * T_{6} * T O O L$, where $B A S E$ and TOOL represent the transformations of base and tool. The positional and orientational components of $P O S, \mathbf{p}_{e}$ and $\left[\mathbf{n}_{e}, \mathbf{o}_{e}, \mathbf{a}_{e}\right]$, represent the position and orientation of the robot's end effector in the Cartesian coordinates. For general consideration, the end-effector will have a length $h_{x}$ in the $\mathbf{n}_{e}$ direction,

Table I. Classification of the primary joints.

\begin{tabular}{cccc}
\hline Case & J1 & J2 & J3 \\
\hline 1 & R & R & R \\
2 & R & R & P \\
3 & R & P & R \\
4 & P & R & R \\
5 & R & P & P \\
6 & P & R & P \\
7 & P & P & R \\
8 & P & P & P \\
\hline
\end{tabular}


another $h_{y}$ in the $\mathbf{o}_{e}$ direction, and the other $h_{z}$ in the $\mathbf{a}_{e}$ direction. With these lengths defined for TOOL, the wrist position $\mathbf{p}_{w}$ can be derived from $P O S$ by the following equation:

$$
\mathbf{p}_{w}=\mathbf{p}_{e}-h_{x} \mathbf{n}_{e}-h_{y} \mathbf{o}_{e}-h_{z} \mathbf{a}_{e}
$$

Equation (5) indicates that $\mathbf{p}_{w}$ depends not only upon the end effector's position but also on its orientation along the planned path. Consequently, the wrist path will not be a straight line even if the end effector's path is a straight line. Due to this orientational effect, the wrist path is unknown until the intermediate orientations are planned.

With $\mathbf{p}_{w}$, the analysis of $P W K$ is performed for the eight combinations of the primary joints listed in Table I. It can be seen that the combinations involving revolute joints are more complicated to analyze than those involving prismatic joints. That is because the prismatic joint moves the link along a straight line, while the revolute joint along a curve. For instance, the workspace of a prismatic and revolute joint with their corresponding links operating in the same plane is bounded by two straight lines and two curves, but that of two revolute joints is bounded by five circles determined by the joint ranges and limits of these two joints. Figure A2 of the Appendix shows one example for the workspace of two revolute joints. Therefore, RRR type in Table I is the most complicated case. In addition, the remaining seven cases can be analyzed following similar and simplified procedures for RRR type with some of the joints substituted by prismatic joint(s). In the analysis of RRR type, to be nonredundant, the joints cannot be all connected in parallel. It will be further divided into three subcases: (a) last two joints in parallel, (b) first two joints in parallel, and (c) all three joints consecutively perpendicular.

In case (a), the last two joints are in parallel as shown in Figure 1. Since the last two joints are in parallel, the traces of the corresponding links will be in the same plane when there are no offsets between these two links. The effects of the offsets to the traces can be identified following the discussion given below.

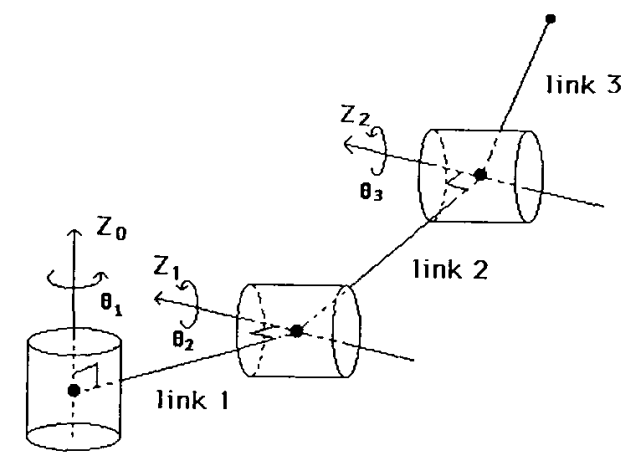

Figure 1. Geometrical structure for last two joints in parallel. 


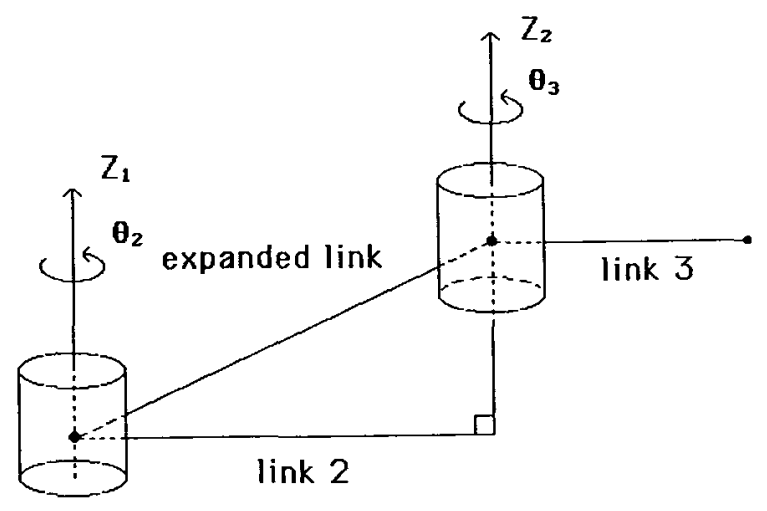

Figure 2. Expanded second link for last two joints in parallel.

By projecting the traces into the plane parallel to the traces and performing the analysis on that plane, the offsets perpendicular to the plane will not have any effect on the projected traces. As for the projections of the offsets parallel to the plane, they will be stationary to the links and can be viewed as part of the links. Figure 2 shows the expanded second link, which consists of the second link and the offset between links two and three. The same reasoning can also be applied to the first link. Similarly, an expanded first link can also be formed. Therefore, with the introductions of the expanded links the effects of offsets are removed. Then the analysis is equivalent to that of a three-link robot manipulator with no offsets. In addition, since the effect of $\theta_{1}$ can be independently identified in this geometry without involving $\theta_{2}$ and $\theta_{3}$ the workspace analysis can be divided into the workspace of joint one and the combined workspace of joints two and three.

In case (b), the first two joints are parallel as shown in Figure 3. Referring to Figure 3, the effect of $\theta_{3}$ can also be independently identified in this geometry without involving $\theta_{1}$ and $\theta_{2}$. Similar to case (a), the analysis can be separated into the workspace of joint three and the combined workspace of joints one and

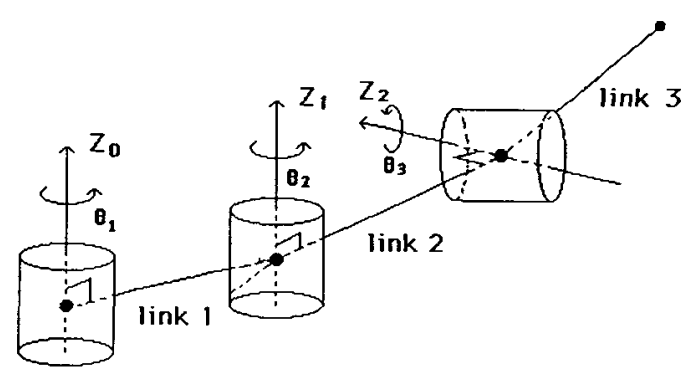

Figure 3. Geometrical structure for first two joints in parallel. 


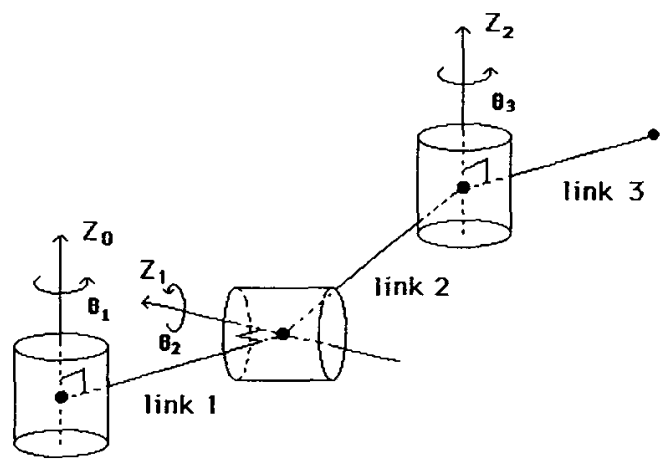

Figure 4. Geometrical structure for three joints consecutively perpendicular.

two. As for case (c), all three joints are consecutively perpendicular as shown in Figure 4. If the effect of $\theta_{2}$ can be identified and removed, the traces of links one and three will be in the same plane. However, the effect from joint two cannot be identified from the wrist position only as in the previous two cases. Nevertheless, if the rotation of joint two can be determined through different approaches or more measured positions, e.g., the end position of link two, the analysis will consist of the workspace of joint two and that of joints one and three.

Based on the discussion above and via proper adjustments, the traces of two of the links will be in the same plane and the trace of the remaining link will be in the plane perpendicular to the aforementioned one. Therefore, there may be two subworkspaces within $P W K$ : One corresponds to a single joint, denoted as $P W K_{1}$, and the other the remaining two joints, denoted as $P W K_{2}$. They are represented as:

$$
P W K_{1}=\left[\mathbf{p}_{1} \mid \mathbf{p}_{1}=\mathbf{f}\left(\theta_{1}\right) \text { for every } \theta_{1}\right]
$$

where $\theta_{1}$ denotes a joint variable of the single joint and $\mathbf{p}_{1}$ the corresponding position, and

$$
P W K_{2}=\left[\mathbf{p}_{2} \mid \mathbf{p}_{2}=\mathbf{f}\left(\boldsymbol{\theta}_{2}\right) \text { for every } \boldsymbol{\theta}_{2}\right]
$$

where $\boldsymbol{\theta}_{2}$ denotes a set of joint variables of the two joints and $\mathbf{p}_{2}$ the corresponding position. As a result, the analysis of $P W K$ can be divided into those of $P W K_{1}$ and $P W K_{2}$. A brief summary of how to generate $P W K_{1}$ and $P W K_{2}$ for case (1) of RRR type is given in the Appendix.

\subsection{Orientational Workspace}

To analyze $O W K$, the orientational part of the point on a Cartesian path, $R_{e}=\left[\mathbf{n}_{e} \mathbf{o}_{e} \mathbf{a}_{e}\right]$, is employed and represented as 


$$
R_{e}=R_{w} *{ }^{w} R_{e}
$$

As indicated in eq. (8), $R_{w}$ in $O W K^{p}$ serves as a reference for mounting ${ }^{w} R_{e}$. Since $R_{w}$ can be determined after the analysis of $\mathbf{p}_{w}$ in $P W K$, the effect from $O W K^{p}$ can be removed and ${ }^{w} R_{e}$ solved. The details of how to solve ${ }^{w} R_{e}$ can be referred to $\mathrm{Wu}$ and Young. ${ }^{10}$ Consequently, the analysis of $O W K$ becomes that of $O W K^{m}$. To analyze $O W K^{m}$, the robot end effector has to be mounted upon the wrist. If the length of the end effector exists in only one direction, then only the rotations of 2 dof can be identified since the rotation of 1 of 3 dof will be redundant. Therefore, the end effector has to consist of lengths at least in two different directions to identify $O W K^{m}$ up to 3 dof. With the end-effector position constrained by the limits and ranges of the minor joints, the analysis of $O W K^{m}$ is equivalent to that of the positional feasibility of the end effector.

To be nonredundant, the geometry of the minor joints has to be consecutively perpendicular. Figure 5 shows one of the connections of the minor joints. To specify the orientation of 3 dof without losing the generality, the tool consists of two lengths, $h_{z}$ in the direction of $\mathbf{a}_{e}$ and $h_{y}$ in the direction of $\mathbf{o}_{e}$. The effect of orientation on these two lengths can be represented in the wrist coordinates $\left(x_{w}, y_{w}, z_{w}\right)$ by the following two equations, respectively:

$$
\begin{aligned}
& { }^{w} \mathbf{h}_{y}=\left[{ }^{w} h_{y_{x}}{ }^{w} h_{y_{y}}{ }^{w} h_{y_{z}}\right]^{t}=h_{y}{ }^{w} \mathbf{o}_{e} \\
& { }^{w} \mathbf{h}_{z}=\left[{ }^{w} h_{z_{x}}{ }^{w} h_{z_{y}}{ }^{w} h_{z_{z}}\right]^{t}=h_{z}{ }^{w} \mathbf{a}_{e}
\end{aligned}
$$

where ${ }^{w} \mathbf{n}_{e},{ }^{w} \mathbf{o}_{e}$, and ${ }^{w} \mathbf{a}_{e}$ are the three orientational vectors of ${ }^{w} \boldsymbol{R}_{e}$.

Referring to Figure 5, the workspace of the tool length $h_{z}$ can only be constrained by the ranges and limits of joints four and five, and joint six has no effect on it. Therefore, ${ }^{w} h_{z}$ can be used to analyze the workspaces of joints four and five. Because the rotations of these two joints are not about the same axis, two subworkspaces are formed corresponding to joints four and five, respectively. On the other hand, the workspace swept by the length $h_{y}$ is constrained by all three joints; however, after the analyses of the workspaces of joints four and five their effects can be removed and ${ }^{w} h_{y}$ can be used to analyze the workspace of joint six alone. As a result, there will be three subworkspaces inside $O W K$. A brief summary of how to generate these subworkspaces is given in the Appendix.

\section{PATH MODIFICATION}

Based upon the above analyses of $P W K$ and $O W K$, the geometric information between a planned path and the workspaces can be derived. To store and exploit the information for path modification, proper geometric indices, denoted as $G I$, are needed. The indices will include the statuses of feasible regions, configurations, and singular areas. To specify these statuses, the measurements will be taken between the planned path and some reference primitives. ${ }^{10}$ The chosen reference primitives are those that describe the work- 


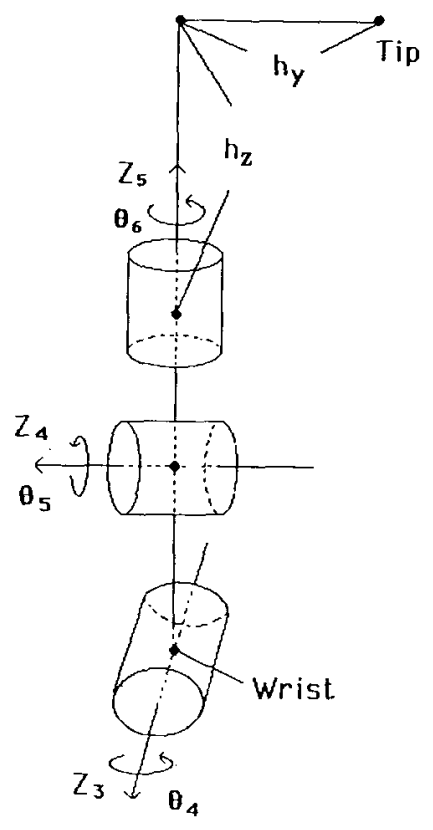

Figure 5. Geometry of tool for orientation feasibility analysis.

spaces and the kinematic constraints, e.g., boundaries of the feasible regions. As a result, a database containing the geometric information along the planned path is ready for modifying those infeasible portions of the traces in the workspaces. Then, path modification can be performed by properly utilizing GI and modification strategies. For evaluation, a successful path modification is defined as:

Definition 1. Successful Path Modification. For a given path, modify its corresponding traces in $\mathrm{PWK}_{1}, \mathrm{PWK}_{2}$, and $\mathrm{OWK}^{\mathrm{m}}$ by appropriately utilizing GI so that the resultant path will be inside the feasible regions of the positional and orientational workspaces with proper configurations and away from the singular areas.

To have successful path modification under different task requirements, modification strategies will vary accordingly. In other words, modification strategies depend upon the properties of the tasks, consequently the freedoms available for modification. For those tasks with the position (orientation) limitation, the freedom in the orientation (position) can be utilized for modification. If only the starting and end points have to be considered, then both the freedoms in position and orientation can be accounted for modification. As for the case in which both the position and orientation specifications have to be satisfied, only feasibility can be checked since there is no freedom for modification. In sum- 
mary, distinguished by the freedoms available, there can be three kinds of modification strategies: position, orientation, and position and orientation modifications.

Since only the kinematic constraints are considered and there is no obstacle involved in the scope of this article, feasible paths always exist between two feasible points due to the continuity of the workspace. However, the statement is no longer true when certain constraints due to the task requirements are imposed. In fact, it is more difficult to satisfy the position than orientation specification. The necessary and sufficient condition for fulfilling the position specification is that every specified position has to be with at least one feasible wrist position. ${ }^{12}$ On the other hand, because most of the industrial robots have quite large joint ranges for the minor joints, the orientation specification can usually be satisfied. For instance, PUMA 560 has total range of joint four $280^{\circ}$, five $200^{\circ}$, and six $532^{\circ}$. However, in order not to hit itself one of the minor joints cannot be designed to have joint range larger than $360^{\circ}$, e.g., joint five in Figure 5. This limitation generates the infeasibility for orientation.

\subsection{Position Modification}

In this case, the orientation specification has to be satisfied. Thus, only the freedom in position can be utilized for modification. As mentioned before, it is usually feasible for $O W K$. Therefore, the proposed scheme will first modify the traces in $P W K$ to obtain feasible wrist positions. Then based on the new $R_{w}$ and the specified $R_{e}$ along the planned path, the new traces in $O W K^{m}$ can be derived. Consequently, the modified planned path in the Cartesian space can be found utilizing these traces. An algorithm for the position modification is as follows:

Position Modification Algorithm. Maintain the orientation specification by modifying the position.

Step 1. Find the wrist position $\mathbf{p}_{w}$. Obtain its corresponding trace in $\mathrm{PWK}_{1}$, denoted as $\operatorname{tr}_{1}^{p}$.

Step 2. Modify $\operatorname{tr}_{1}^{\mathrm{p}}$ in $\mathrm{PWK}_{1}$ to be within feasible regions when infeasible, denoted as ${ }^{n} \operatorname{tr}_{1}^{\mathrm{p}}$, and ${ }^{\mathrm{n}} \mathrm{tr}_{1}^{\mathrm{p}}$ corresponds to a set of joint variables, denoted as $\mathrm{q}_{\mathrm{p}}^{\mathrm{p}}$. Note here that according to the analysis in the Appendix $\operatorname{tr}_{1}^{\mathrm{P}}$ is always feasible when the range of the single joint is larger than $180^{\circ}$. When the joint range is less than $180^{\circ}$, the modification will utilize the measurement in GI to move the infeasible portion to be within feasible regions.

Step 3. Obtain a new trace $\operatorname{tr}_{2}^{\mathrm{p}}$ in $\mathrm{PWK}_{2}$ utilizing ${ }^{n}{ }^{\mathrm{t}}{ }_{1}^{\mathrm{p}}$ and $\mathrm{PW}_{\mathrm{z}}$ of the original wrist position $\mathrm{Pw}$. Modify $\operatorname{tr}_{2}^{\mathrm{p}}$ to be within the feasible regions, denoted as ${ }^{\mathrm{n}} \operatorname{tr}_{2}^{\mathrm{p}}$. According to the distance between the trace and feasible regions stored in GI, possible modification can be to move the infeasible portion within the boundary of the feasible region or just connect the starting and end positions with an arbitrary feasible trace. Note that the modification of $\operatorname{tr}_{2}^{\mathrm{p}}$ to ${ }^{\mathrm{n}} \mathrm{tr}_{2}^{\mathrm{p}}$ will deviate 
${ }^{n_{1}}{ }_{1}^{p}$ to another new trace ${ }^{\mathrm{n}_{\mathrm{n}}} \operatorname{tr}_{1}^{\mathrm{p}}$, while ${ }^{\mathrm{n}_{\mathrm{n}}} \mathrm{r}_{1}^{\mathrm{p}}$ will still correspond to the same set of joint variables $\mathrm{q} \mathrm{p}$.

Step 4. Find the new wrist orientation ${ }^{n} R_{w}$ corresponding to ${ }^{n_{n}} t_{1}^{p}$ and ${ }^{n} \operatorname{tr}_{2}^{p}$. Then, obtain the new traces $\operatorname{tr}^{\mathrm{m}}$ in $\mathrm{OWK}^{\mathrm{m}}$ utilizing ${ }^{\mathrm{w}} \mathrm{R}_{\mathrm{e}}$ derived from ${ }^{\mathrm{n}} \mathrm{R}_{\mathrm{w}}$ and $\mathrm{R}_{\mathrm{e}}$ along the planned path. Check the feasibility of $\operatorname{tr}^{\mathrm{m}}$. If it is infeasible, find another feasible wrist position via Steps 2 and 3 and perform Step 4 again.

Step 5. Find the corresponding path in the Cartesian space using these five newly derived traces in the workspaces.

When $t r^{m}$ in Step 4 is not feasible, the procedure may be performed several times in the process of finding feasible $\mathrm{tr}^{\mathrm{m}}$. As most of the industrial robot manipulators have quite large joint ranges for minor joints, it is a rare case.

\subsection{Orientation Modification}

In this case, the position specification has to be satisfied. Therefore, only the freedom in orientation can be utilized for modification. Unlike the position modification in the previous section, it is not uncommon that modification is not possible. As aforementioned, the sufficient and necessary condition for possible modification is that each of the specified positions has to be with at least one feasible wrist position. It means that the corresponding feasible wrist position can reach the specified position with feasible orientation(s). ${ }^{12}$ An algorithm for orientation modification is given below:

Orientation Modification Algorithm. Maintain the position specification by modifying the orientation.

Step 1. Find the point $\mathrm{p}$ on the infeasible portion that is "most" infeasible, defined as its corresponding locations in $\mathrm{PWK}_{1}$ and $\mathrm{PWK}_{2}$ most away from feasible regions.

Step 2. Maintain $\mathbf{p}_{e}$ of $\mathrm{p}$ and modify its $\mathrm{R}_{\mathrm{e}}$ to become a feasible point ${ }^{\mathrm{n}} \mathrm{p}$. If no feasible $\mathrm{R}_{\mathrm{e}}$ can be found, modification is impossible and exit. Otherwise, replan the orientation of the infeasible portion by interpolating the orientational parts of ${ }^{\mathrm{n}} \mathrm{p}$ and neighboring feasible points.

Step 3. If the replanned path is feasible, the modification is successful and exit. Otherwise, identify the infeasible portions and go to Step 1.

\subsection{Position and Orientation Modification}

In this case, both the freedoms in position and orientation can be utilized for path modification. By treating the infeasible regions constrained by the kinematics as obstacles, the problem can be viewed as that of obstacle avoidance inside the robot workspace. 


\section{SIMULATIONS}

To demonstrate the proposed feasibility and modification schemes, a simple robot task is simulated. The task is to move robot's tool-tip from point $(-0.6$, $0.5,1.1)$ to $(0.4,0.4,1.9)$ along a straight line with a fixed orientation. The $6 \mathrm{R}$ robot manipulator with no offset in Figure 6 is selected for simulation. The joint ranges chosen for this robot are similar to those of industrial robots. The kinematic specifications for the robot manipulator are set as: $a_{2}=0.6 \mathrm{~m}, d_{1}=$ $1.2 \mathrm{~m}$, and $d_{4}=0.4 \mathrm{~m}$. Since there are 3 dof for the minor joints, tool lengths are incorporated in both $\mathbf{o}_{e}$ and $\mathbf{a}_{\ell}$ directions and set as $h_{y}=0.1 \mathrm{~m}$ and $h_{z}=0.2 \mathrm{~m}$. The ranges of joints one to six are $\left(-60^{\circ}, 135^{\circ}\right),\left(-135^{\circ}, 135^{\circ}\right),\left(-135^{\circ}, 0^{\circ}\right)$, $\left(-60^{\circ}, 200^{\circ}\right),\left(-50^{\circ}, 150^{\circ}\right)$, and $\left(-200^{\circ}, 200^{\circ}\right)$ respectively.

Based upon the discussions in the Appendix, the given path is mapped into the traces in $P W K$ and $O W K$ as shown in Figures 7 and 8 . Within $P W K$, the workspace corresponding to joint one is referred to as $P W K_{1}$, and that for joints two and three $P W K_{2}$. Within $O W K$, there are three subworkspaces corresponding to three minor joints. The solid lines in Figure 7 show two traces in $P W K$ and in Figure 8 three traces in $O W K$. In Figure 7, the trace in $P W K_{2}$ is not

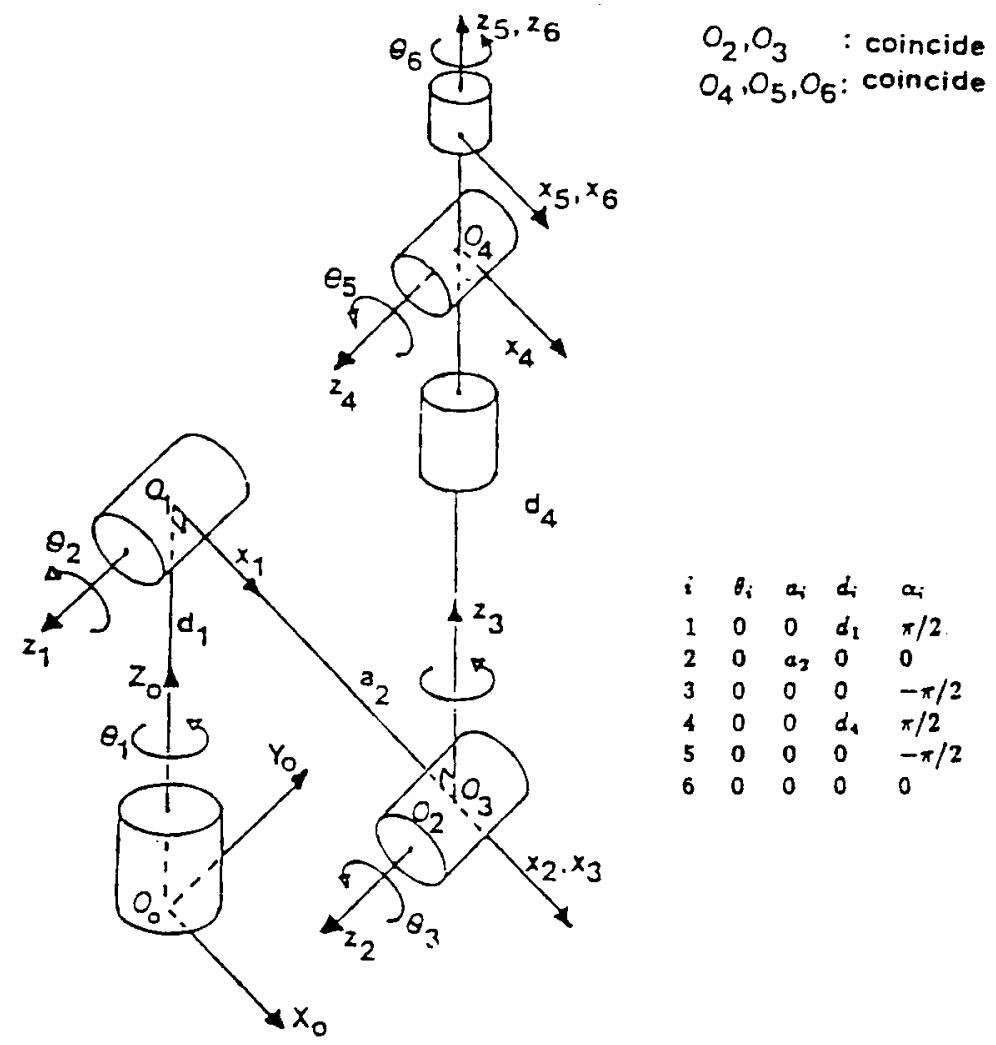

Figure 6. 6R manipulator mechanism. 

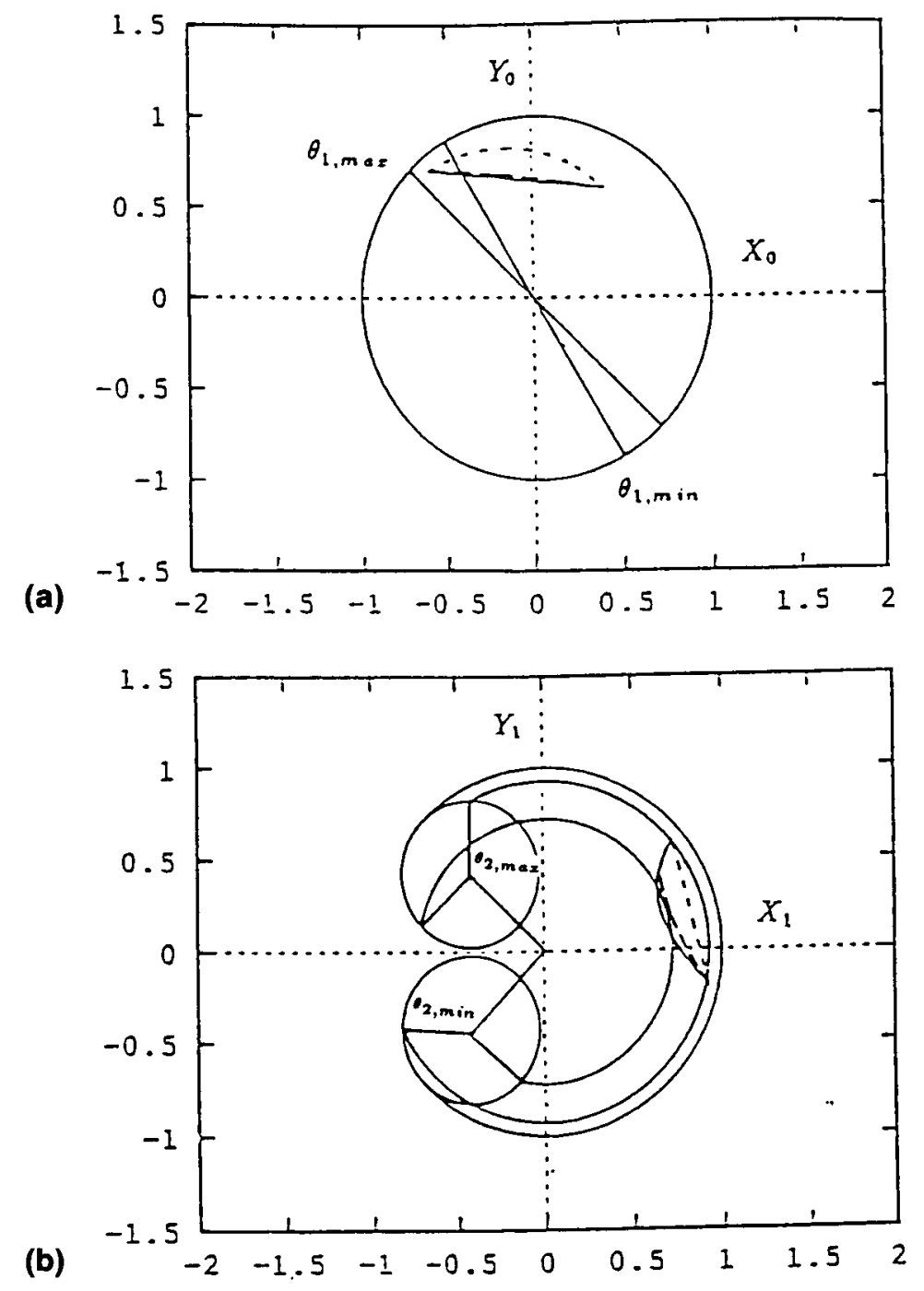

Figure 7. (a) Traces in the positional workspace before and after position and orientation modifications: joint one workspace. (b) Joints two and three workspace.

feasible due to that part of it that is out of the feasible region described in the Appendix. First, a simulation is executed for the position modification to maintain the fixed orientation along the path. Following the position modification algorithm in the previous section, the modification in $P W K_{2}$ is to replace the infeasible trace with a feasible straight line, shown as the short dotted lines in Figure $7 \mathrm{~b}$. The new trace in $P W K_{1}$ due to the modification in $P W K_{2}$ is as the short dotted line in Figure 7a. Utilizing the new wrist coordinates obtained from these two new traces along with the specified orientations of the given 
(a)

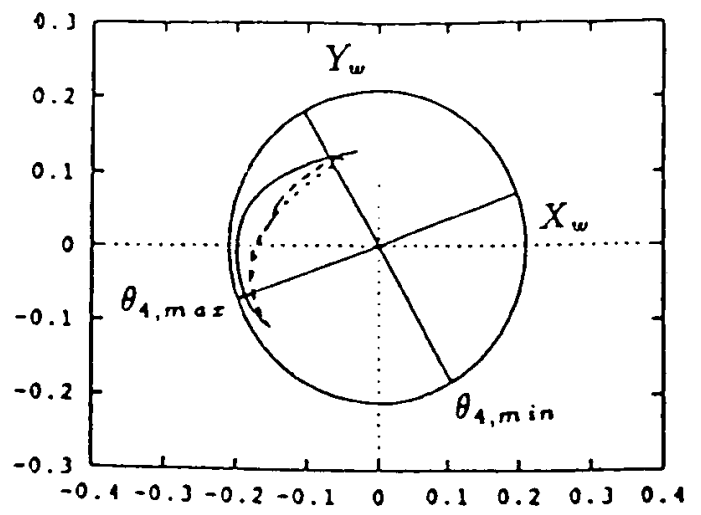

(b)

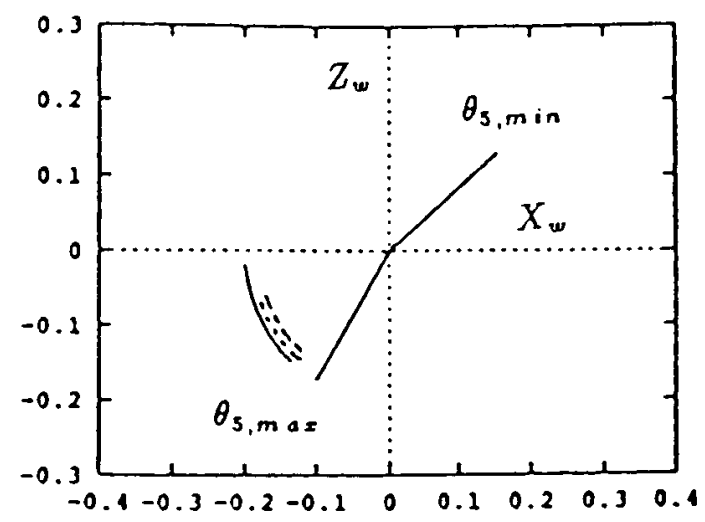

(c)

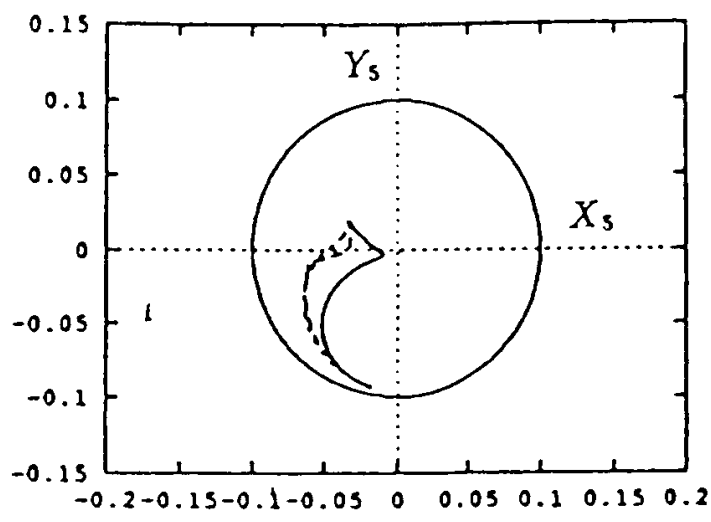

Figure 8. (a) Traces in the orientational workspace before and after position and orientation modifications: joint four workspace. (b) Joint five workspace. (c) Joint six workspace. 


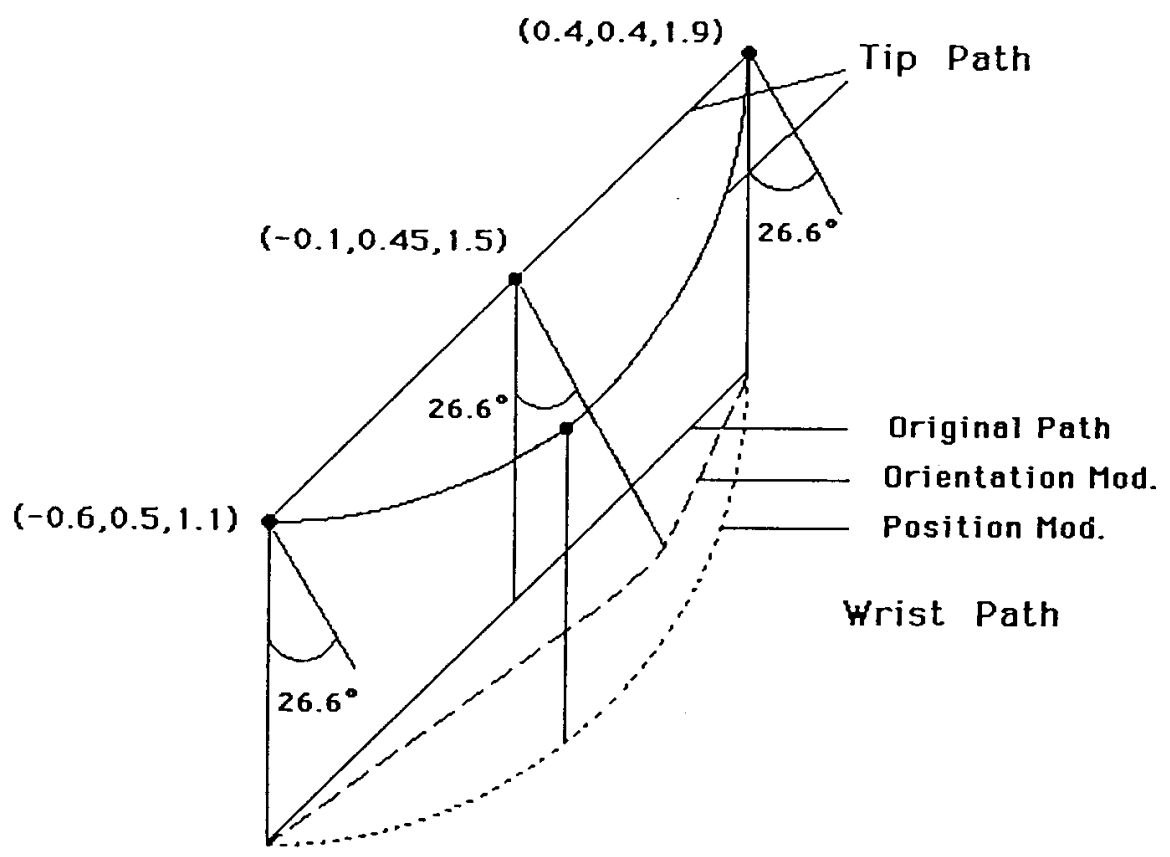

Figure 9. Tip and wrist paths before and after position and orientation modifications.

path, the new traces in $O W K$ can be obtained and are shown as the short dotted lines in Figures $8 \mathrm{a}-\mathrm{c}$. They are feasible and do not need modification. The resultant modified tip and wrist paths overlaying with the original paths are shown in Figure 9. This modification scheme modifies an infeasible straight-line path into a feasible curved one while it maintains the original orientation.

Another simulation is executed to demonstrate the orientation modification. The modification will maintain the original positions along the path by modifying the orientations. Following the orientation modification algorithm, point $(-0.1,0.45,1.5)$, referred to as point $\mathrm{A}$, is found to be most infeasible. It is modified to be a feasible point ${ }^{n} A$ by rotating about $x$-axis $26.6^{\circ}$ with the length of end effector, $\left(0.1^{2}+0.2^{2}\right)^{1 / 2}$, as the radius, and point $\mathrm{A}$ as the center. The modification maintains the position of point $A$ and moves its wrist position to be within feasible regions. Different rotation axes and angles can also be adopted if only a feasible wrist position can be obtained. With the starting, ${ }^{n} A$, and end points, the orientation of the path is replanned by interpolating their orientational parts. The resultant trace in $P W K_{2}$ is feasible and needs no more modification, shown as the long dotted line in Figure $7 \mathrm{~b}$. The new trace in $P W K_{1}$ via the replanning is shown as the long dotted line in Figure $7 \mathrm{a}$ and those in $O W K$ as the long dotted lines in Figures $8 \mathrm{a}-\mathrm{c}$. They are feasible and do not need modification. The resultant modified tip and wrist paths are shown in Figure 9. This modification scheme maintains the original straight-line path while the orientation is modified. Consequently, the original straight-line wrist path is modified to be a curved one. 


\section{CONCLUSIONS}

In this article, schemes for kinematic feasibility testing and modification are proposed. Via the geometrical analysis, proper geometric expressions describing the robot workspace and the kinematic constraints are derived. Furthermore, based on different task requirements, corresponding modification strategies are proposed. By utilizing the derived geometrical information and the proposed modification strategies, a kinematically infeasible path can be modified accordingly. The simulation results verify the proposed schemes. In addition, it shows that the geometric workspace analysis can provide amply information for path planning due to the picturesqueness of geometric demonstration. Consequently, the integration between $\mathrm{CAD} /$ robots can be enhanced by proper utilization of the analysis.

\section{References}

1. R. P. Paul, Robot Manipulators: Mathematics, Programming, and Control, MIT Press, Cambridge, MA, 1981.

2. A. Kumar and K. J. Waldron, "The dexterous workspace," Trans. ASME, paper, 80-DET-108, 1980.

3. D. C. H. Yang and Z. C. Lai, "On the dexterity of robotic manipulators, service angle," ASME J. of Mechanics, Transmission, and Automation in Design, 262$270(1985)$.

4. Y. C. Tsai and A. H. Soni, "Accessible region and synthesis of robot arms," ASME J. of Mechanical Design, 103, 803-811, (1981).

5. J. A. Hansen, K. C. Gupta, and S. M. K. Kazerounian, "Generation and evaluation of the workspace of a manipulator," Int. J. of Robotics Research, 2(3), 22-31 (1983).

6. T. W. Lee and D. C. H. Yang, "On the evaluation of manipulator workspace," Trans. of ASME, J. of Mechanics, Transmission, and Automation in Design, 1-8, (1983).

7. F. L. Litvin, Z. Yi, V. P. Castelli, and C. Innocenti, "Singularities, configurations, and displacement functions for manipulators," Int. J. of Robotics Research, 5(2), 52-65 (1986).

8. R. Vijaykumar, M. J. Tsai, and K. J. Waldron, "Geometric optimization of serial chain manipulator structures for working volume and dexterity," Int. J. of Robotics Research, 5(2), 91-103 (1986).

9. C. S. G. Lee and M. Ziegler, "A geometric approach in solving the inverse kinematics of PUMA robots," IEEE Trans. on Aerospace and Electronics Systems, 20(6), 695-706 (1984).

10. C. H. Wu, and K. Y. Young, "Robot workspace geometry for trajectory feasibility study," Proc. of IEEE International Conf. on Systems, Man, and Cybernetics, 1988, pp. 238-241.

11. J. C. Colson and N. D. Perreira, "Kinematic arrangements used in industrial robots," Proc. of 13th ISIR, 1983, pp. 20:1-18.

12. C. C. Jou, "On the orientational feasibility of robot manipulators," Proc. of International Conf. on Automation, Robotics, and Computer Vision, 1990, pp. 524528 .

\section{APPENDIX}

The following is a summary of how to generate the workspace of the $6 \mathrm{R}$ robot manipulator with no offsets in Figure 6. The wrist position $\mathbf{p}_{w}$ in eq. (5) is 
utilized to generate the positional workspace, and ${ }^{w} h_{y}$ and ${ }^{w} h_{z}$ in eqs. (9) and (10) the orientational workspace. The reasoning and detailed derivation can be referred to $\mathrm{Wu}$ and Young. ${ }^{10}$

\section{A. Workspace of Joint One}

By projecting the wrist position $\mathbf{p}_{w}$ onto the $\left(x_{0}, y_{o}\right)$ plane, the joint one workspace can be obtained as shown in Figure A1. Referring to Figure A1, the workspace is divided into four areas by two lines, $\mathbf{l}_{1}$ and $\mathbf{I}_{2}$, which correspond to the projections of $\mathbf{p}_{w}$ when the positions of joint one are maximum and mini-

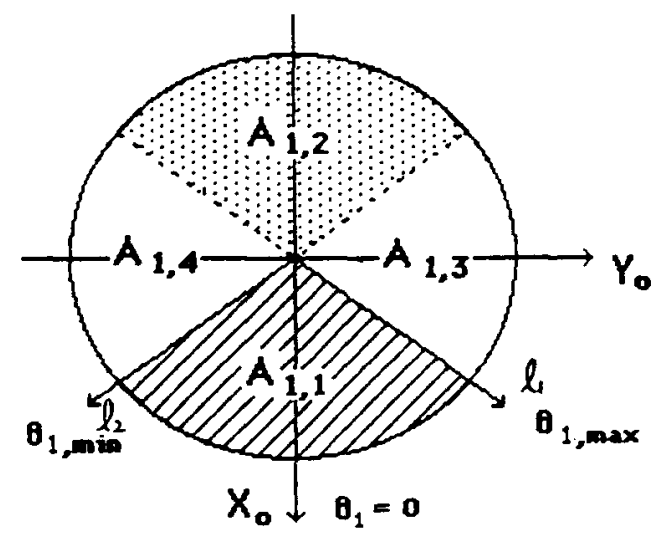

(a) Ronge $\left(\theta_{1}\right) \leq 180^{\circ}$.

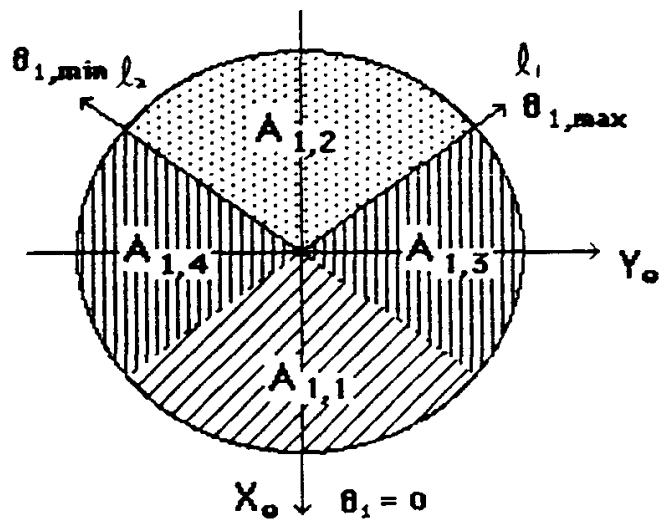

(b) Range $\left(\theta_{1}\right)>180^{\circ}$.

Figure A1. Workspace of a single joint $\left(P W K_{1}\right)$. 
mum, respectively. There are two possible configurations in this workspace, referred to as forward and backward arm configurations. The configuration of a backward arm is defined as that of a forward arm by adding the position of joint one $180^{\circ}$ and adjusting other joints to reach the same position.

Figure Ala shows the case of range $\left(\theta_{1}\right) \leq 180^{\circ}$. When the joint one workspace is divided by $\mathbf{I}_{1}$ and $\mathbf{I}_{2}$ in this way, the projections on $A_{1,1}$ and $A_{1,2}$ will correspond to joint one within the joint limit. They can only be reached by the configuration of a forward arm since range $\left(\theta_{1}\right) \leq 180^{\circ}$. The projections on $A_{1,3}$ and $A_{1,4}$ will correspond to joint one out of the joint limit and cannot be reached by the given robot. On the other hand, in the case of range $\left(\theta_{1}\right)>180^{\circ}$ shown in Figure Alb the projections on $A_{1,1}$ and $A_{1,2}$ are still feasible with the forward arm configuration. However, the projections on $A_{1,3}$ or $A_{1,4}$ are feasible with both configurations due to range $\left(\theta_{1}\right)>180^{\circ}$. Except the vicinity of the boundary of the feasible regions, the singular area also includes the origin $O_{0}$, where infinite solutions of joint one can be found along this line.

\section{B. Workspace of Joints Two and Three}

With the effect from joint one removed following the analysis of the joint one workspace, the joint two and three workspace can be obtained by rotating joints two and three from their minimums to maximums. Due to the geometries of joints two and three, the boundary of the joint two and three workspace depends not only upon the limits but also the range of $\theta_{3} .{ }^{10}$ There are four different types of workspaces corresponding to different ranges of $\theta_{3}$ : $\left|\theta_{3, A B}\right| \leq\left|\theta_{3, B C}\right|,\left|\theta_{3, A B}\right| \geq\left|\theta_{3, B C}\right|, \theta_{3} \leq-90^{\circ}$, and $\theta_{3} \geq-90^{\circ}$. Figure A2 shows the

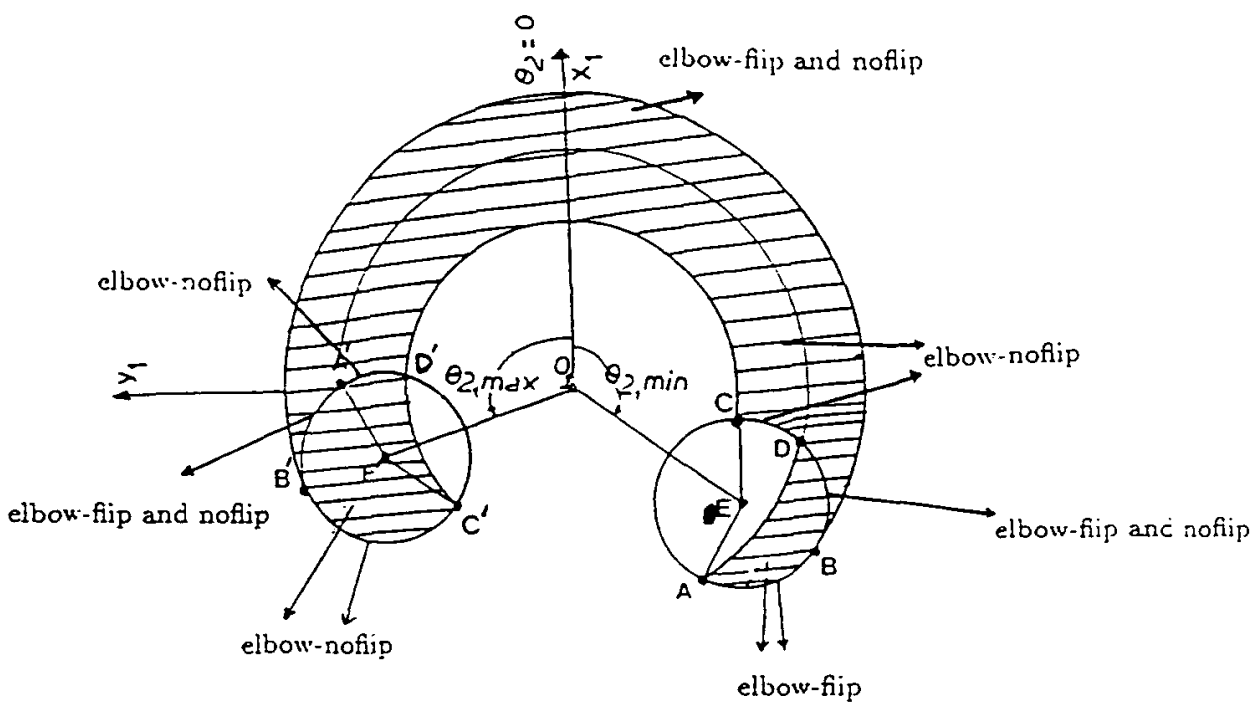

Figure A2. Workspace of two joints $\left(P W K_{2}\right)$. 


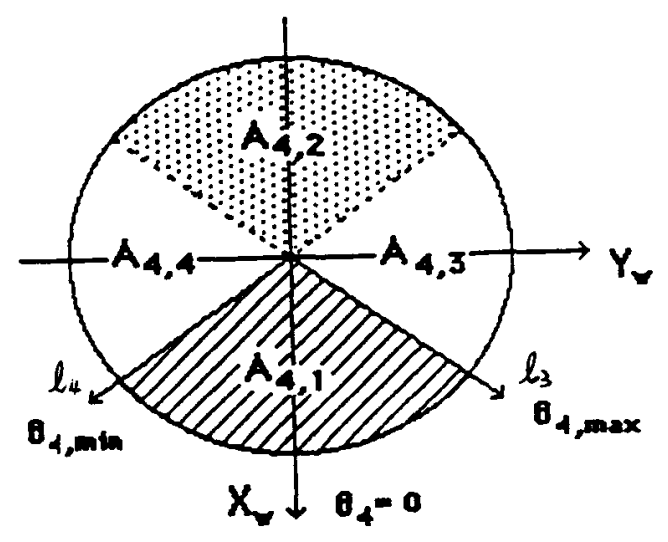

(a) Range $\left(\theta_{4}\right) \leq 180^{\circ}$.

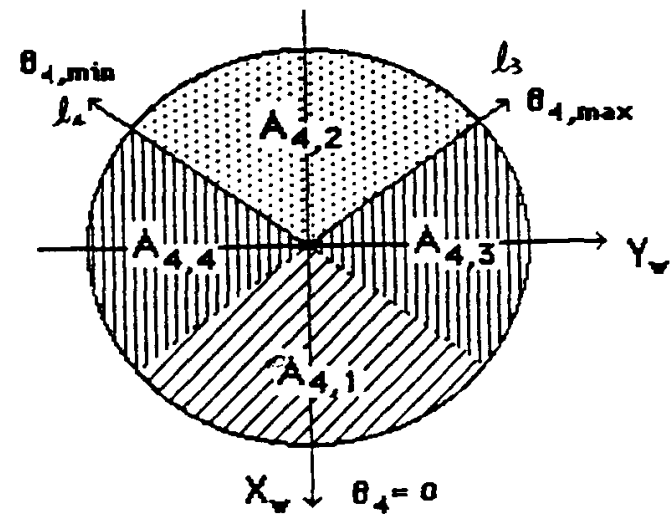

(b) Renge $\left(\theta_{4}\right)>180^{\circ}$.

Figure A3. Workspace of joint four.

case that $\left|\theta_{3, A B}\right| \leq\left|\theta_{3, B C}\right|$, where point $O_{1}$ is the origin of link 2; points $E$ and $E^{\prime}$ correspond to the origins of link 3 , when $\theta_{2}$ is at its minimum and maximum, respectively; points $C$ and $C^{\prime}$ correspond to $\theta_{3, \max }$; and points $A$ and $A^{\prime}$ correspond to $\theta_{3, \min }$.

There are two configurations in the joint two and three workspace, referred to as elbow-nonflip and elbow-flip, depending upon the position of joint three. For the given robot, elbow-nonflip is defined as when $-90^{\circ} \leq \theta_{3} \leq 90^{\circ}$; while for other positions of $\theta_{3}$ the arm is with the configuration of elbow-flip. The areas of different elbow configurations are analyzed and identified as shown in Figure A2. 


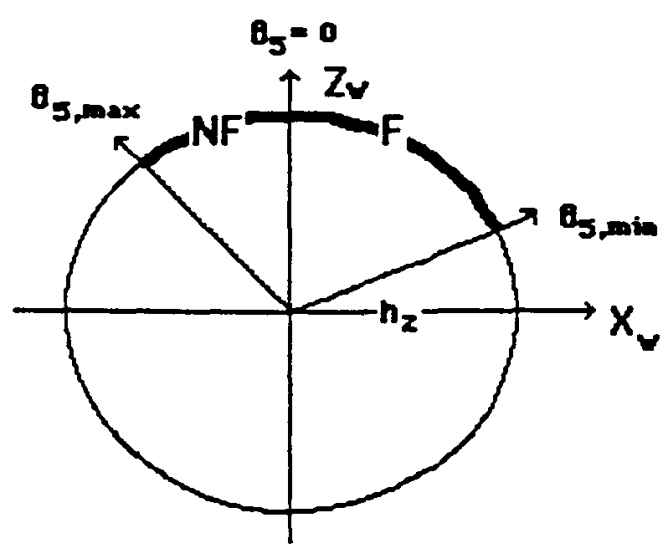

(a) Range $\left(\theta_{5}\right) \leq 180^{\circ}$.

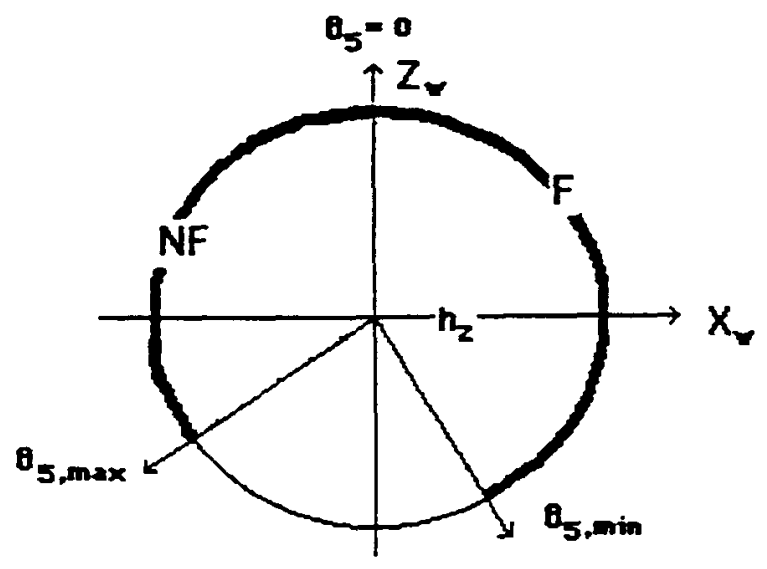

(b) Renge $\left(\theta_{5}\right)>180^{\circ}$.

Figure A4. Workspace of joint five.

\section{Workspaces of Joints Four, Five, and Six}

Similar to the procedures to analyze the joint one workspace, the joint four workspace can be obtained by projecting ${ }^{w} \mathbf{h}_{z}$ in eq. (10) onto the $\left(x_{w^{\prime}}, y_{w^{\prime}}\right)$ plane as shown in Figure A3. The workspace is also divided into four areas by two lines, $\mathbf{l}_{3}$ and $\mathbf{l}_{4}$, which correspond to the projections of " $h_{z}$ when the positions of joint four are maximum and minimum, respectively. The areas of different configurations and the singular area can also be determined similar to the derivation for the joint one workspace. 


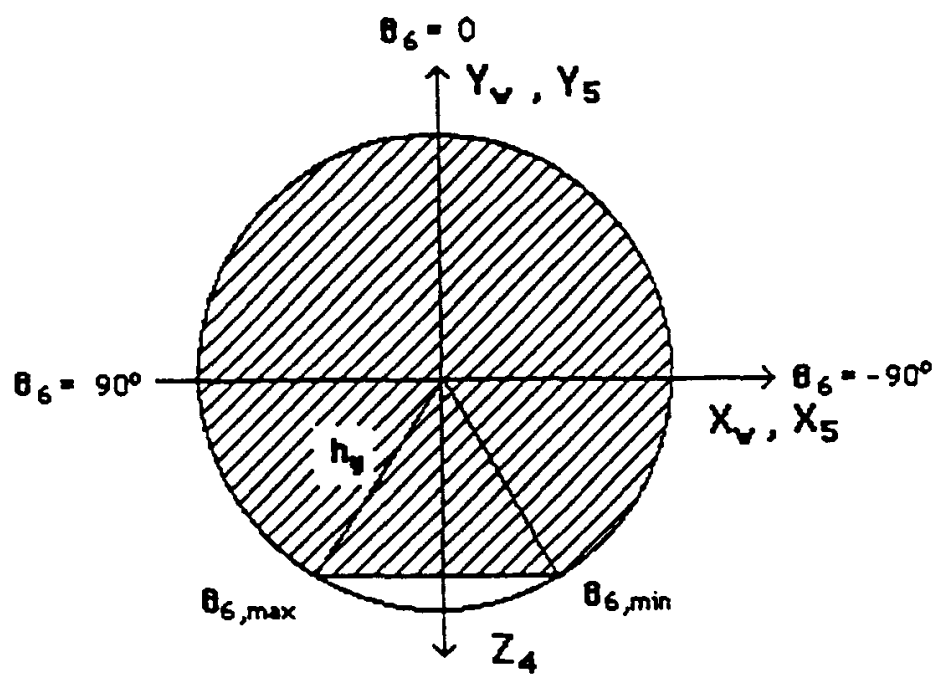

Figure A5. Workspace of joint six.

After the analysis of the joint four workspace, a new vector " $\mathbf{h}_{z}^{\prime}$ on the plane of $\left(x_{w}, z_{w}\right)$ can be obtained by removing the effect from joint four. Then, the trace of ${ }^{w} h_{z}^{\prime}$ is governed by joint five only. Consequently, the joint five workspace is a curve with the radius of length $h_{z}$ and bounded by the limit of joint five as shown in Figure A4.

After analyses of the workspaces of joints four and five, ${ }^{w} \mathbf{h}_{y}$ in eq. (9) will be used to analyze the joint six workspace. To simplify the analysis, the length $h_{z}$ will be assumed to be zero since the feasibility of ${ }^{w} \boldsymbol{h}_{z}$ has been tested. First, consider a case in which $\theta_{4}=0^{\circ}, \theta_{5}$ with full range of $360^{\circ}$, and $\theta_{6, \max }=-\theta_{6, \min }$, i.e., the joint six workspace is symmetric to $y_{w}\left(y_{5}\right)$ axis. Then, all the projections of feasible ${ }^{w} h_{y}$ on the $\left(x_{w}, y_{w}\right)$ plane will form the shaded area shown in Figure A5. To have the condition in which $\theta_{4}=0$, the effect of joint four is removed from ${ }^{w} h_{y}$ by rotating the vector $-\theta_{4}$ about the axis $z_{w}$ to be ${ }^{w} \mathbf{h}_{y}^{\prime}$. To satisfy the second condition, a new vector " $h_{y}^{\prime \prime}$ is obtained by rotating " $\mathbf{h}_{y}^{\prime}$ about ${ }^{w} z_{5}$ axis an angle of $-\left(\theta_{6, \min }+\theta_{6 . \max }\right) / 2$. After these two transformations, the projection of "h" $h_{y}^{\prime \prime}$ on the $\left(x_{w}, y_{w}\right)$ plane can then be used to determine the feasibility of joint six utilizing the workspace shown in Figure A5. 